Itinéraires Itinéraires

Littérature, textes, cultures

2011-1| 2011

Les Mémoires, une question de genre?

\title{
Masculin/Féminin chez George Sand
}

\author{
Béatrice Didier
}

\section{(2) OpenEdition}

Journals

Édition électronique

URL : http://journals.openedition.org/itineraires/1629

DOI : $10.4000 /$ itineraires.1629

ISSN : 2427-920X

Éditeur

Pléiade

Édition imprimée

Date de publication : 1 avril 2011

Pagination : 85-91

ISBN : 978-2-296-13692-2

ISSN : 2100-1340

Référence électronique

Béatrice Didier, « Masculin/Féminin chez George Sand », Itinéraires [En ligne], 2011-1 | 2011, mis en ligne le 01 avril 2011, consulté le 10 décembre 2020. URL : http://journals.openedition.org/itineraires/ 1629 ; DOI : https://doi.org/10.4000/itineraires.1629

Itinéraires est mis à disposition selon les termes de la licence Creative Commons Attribution - Pas d'Utilisation Commerciale - Pas de Modification 4.0 International. 


\title{
Masculin/Féminin chez George Sand
}

\begin{abstract}
In the nineteenth century, the distinction between the masculine and the feminine is clearly displayed in clothing and the distribution of tasks, and George Sand evokes this with humor in Histoire de ma vie. However, her novelistic work seems to clearly mark an evolution with the transformation of the central feminine character: from woman as a victim (Indiana, victim of the husband, the lover, society) to the strong woman (Lélia, Abbess of Camaldules in the 1839 version; Tonine, running a factory in La Ville Noire). However, it is in the dialogue with Flaubert that this relationship between the masculine and the feminine takes its most original form, with the assertion that creation is fundamentally hermaphroditic.
\end{abstract}

Keywords : autobiography, hermaphroditism, Flaubert, the image of the woman, nineteenth century literature

Mots clés : autobiographie, hermaphrodisme, Flaubert, image de la femme, littérature du XIX ${ }^{\mathrm{e}}$ siècle

Je n'entends pas traiter ici du féminisme de George Sand, sujet souvent abordé, et d'ailleurs de façon parfois contestable, parce qu'insuffisamment replacé dans des perspectives historiques : le féminisme de George Sand ne peut par être celui des années 1970. Si je m'inspire pour mon titre de celui de Françoise Héritier que j'admire, c'est aussi parce que mon étude ne voudrait pas être exclusivement littéraire, mais introduire aussi quelques éléments de sociologie dans une analyse de la répartition des sexes.

Je voudrais rappeler d'abord quelques faits historiques bien connus, mais qu'il est nécessaire d'avoir à l'esprit. La Révolution française, puis l'Empire, la Restauration et la monarchie de Juillet ont contribué à renforcer la forte différenciation du masculin et du féminin. On sait avec quelle violence Robespierre s'est insurgé contre toute ingérence des femmes dans le domaine politique considéré comme exclusivement masculin. La femme doit être essentiellement épouse et mère; la société bourgeoise ascendante en ce début du XIX ${ }^{e}$ siècle exalte ces qualités; Napoléon et son Code civil 
renforcent cette différenciation : la femme doit essentiellement donner des enfants qui deviendront des soldats. La restauration du catholicisme va dans le même sens : la femme doit donner à l'Église de nouveaux chrétiens. La vie quotidienne souligne le contraste. Le costume qui, de plus en plus, prive l'homme d'ornements, accentue aussi la différence, tandis que le pantalon est masculin et que son port par une femme fait scandale. C'est ce que George Sand relate dans l'Histoire de ma vie avec humour : « Les hommes portaient de longues redingotes carrées, dites à la propriétaire. » Quelle différence avec les costumes chamarrés (du moins pour les riches) de l'Ancien Régime! L'élégance, les couleurs dans le costume masculin sont désormais mal vus par la morale, et considérés comme un dandysme suspect. Le bourgeois propriétaire est habillé le plus souvent en noir, tandis que le vêtement féminin a le droit de prétendre à l'élégance; encore faut-il pouvoir se la payer. Sand rapporte ce propos de Balzac : «On ne peut pas être femme à Paris à moins d'avoir vingt-cinq mille francs de rente ${ }^{1}$. » Lorsque George Sand adopte un costume masculin, c'est par économie, mais aussi avec un secret plaisir de brouiller les pistes et elle se complaît dans l'ambiguïté. Ainsi chez Pinson où elle prend ses repas tantôt habillée en femme, tantôt habillée en homme, le restaurateur ne sait jamais s'il faut lui dire monsieur ou madame : « Il ne s'était pas plutôt accoutumé à me dire monsieur que je paraissais en femme, et il n'arrivait à dire madame que le jour où je redevenais monsieur ${ }^{2}$. »

La répartition des tâches est aussi strictement établie. Dans ses débuts la jeune Aurore qui n'est pas encore George Sand est réduite à des travaux d'aiguille, elle en découvre l'intérêt lorsqu'elle attend son fils Maurice; dans cette période de grossesse, il lui semble que les facultés intellectuelles s'arrêtent : «Les besoins de l'intelligence, l'inquiétude des pensées, les curiosités de l'étude, comme celles de l'observation, tout disparut dès que le doux fardeau se fit sentir ${ }^{3}$. » Par la suite, d'ailleurs George Sand restera capable de travaux manuels (elle fabrique des costumes pour les marionnettes de Nohant); elle aura dépassé ce clivage qu'elle a fortement subi dans sa première jeunesse, et semble même avoir provisoirement accepté. On remarquera aussi que l'autobiographe note que sa grand-mère ne lui avait pas du tout appris à coudre - détail, me semble-t-il, révélateur, dans la mesure où la grand-mère représente la noblesse d'Ancien Régime, elle représente aussi une société où la femme n'est pas enfermée dans des tâches ménagères et où son accès à l'écriture est beaucoup plus libre.

Le clivage est absolu aussi dans la société louis-philipparde entre l'homme qui fait des livres, et la femme qui fait des enfants : on a souvent

1. George Sand, Histoire de ma vie, éd. G. Lubin, Paris, Gallimard, coll. « Bibliothèque de la Pléiade », t. II, p. 116.

2. Ibid., t. II, p. 119.

3. Ibid., t. II, p. 32. 
cité la fameuse entrevue de Sand et du journaliste Keratry. Après avoir développé sa théorie de l'infériorité des femmes, « il termina sa harangue par un trait napoléonien qui devait m'écraser : "Croyez-moi, me dit-il gravement comme j'ouvrais la dernière porte de son sanctuaire, ne faites pas des livres, faites des enfants" " ». La romancière a aperçu Mme Keratry « couchée sous un couvre-pied de soie rose très galant» qui a jeté « un regard de pitié languissante sur ma robe de stoff et sur mes souliers crottés ${ }^{5} »$.

C'est donc dans ce contexte social que la jeune femme va avoir à s'imposer, et d'abord, comme l'a bien montré Martine Reid, en signant Sand. Le rapport masculin/féminin est évidemment essentiel dans le choix de ce nom d'auteur. Comme dans le choix du vêtement, le rapport masculin/ féminin se double d'une allusion à une différence de classe : George est un prénom d'homme et de paysan; il contient aussi une référence géographique : c'est un prénom berrichon. Cependant des ambiguïtés demeurent, parce que George est un prénom qui peut-être féminin en anglais, et que Aurore a été quelque temps élevée dans un couvent de religieuses anglaises où elle a commencé à écrire. Quant au nom, il est encore plus chargé d'ambiguïtés; apparemment il marque une volonté d'indépendance et de rupture, un désir de la romancière de signer ce qu'elle a écrit et de sortir de ce brouillage de la collaboration avec Jules Sandeau (on se reportera au tome I de l'édition des Euvres Complètes de Sand, qu'a publiée Yves Chastagnaret $\left.{ }^{6}\right)$. Cependant elle n'invente pas totalement, elle reprend le nom d'un homme, mais auquel elle fait subir une coupure, les psychanalystes diront une castration.

Je n'ai évidemment pas l'intention d'analyser ici le rapport du masculin et du féminin, dans la totalité de l'œuvre romanesque de George Sand qui a écrit plus de quatre-vingt-dix romans. Je voudrais simplement consigner quelques remarques. Le premier roman signé Sand, Indiana, malgré ses audaces, sa critique du mariage et de la société, présente dans le personnage éponyme les caractéristiques essentielles de ce que l'on pourrait appeler le féminin romanesque de son époque : Indiana, même si elle a finalement au moins le courage de s'en aller, est faible devant les violences de son mari, faible aussi devant les exigences, puis l'abandon de son amant Raymon; dans la dernière étape de sa vie, dans cette idylle exotique, elle reste silencieuse, et Ralph a le rôle masculin traditionnel de protecteur de la femme victime. Indiana est dépendante psychologiquement et matériellement; il était nécessaire de brosser ce tableau pitoyable pour que la critique des institutions prenne tout son sens; mais Indiana n'est pas George Sand, quoique les critiques aient toujours tendance à assimiler l'héroïne et la romancière, surtout quand elle est jeune. George Sand marque une forte

4. George Sand, op. cit., t. II, p. 150.

5. Ibid., t. II, p. 148.

6. George Sand avant Indiana, éd. Yves Chastagnaret, dans Béatrice Didier (dir.), Euvres complètes, Paris, Champion, 2008. 
distance par rapport à son modèle grâce aux ressources de la voix narrative : le narrateur est sévère pour Indiana, et le narrateur est un homme : autre façon de déprécier l'héroïne? façon surtout de montrer que la romancière est capable de distance, refuse de s'enfermer dans ce féminin minoré, est capable d'emprunter une voix narrative masculine. La deuxième version, celle de 1833, parce qu'elle réduit les interventions du narrateur rend moins nette cette distanciation qui subsiste cependant. Geneviève est aussi une héroïne très féminine au sens où on l'entend alors; néanmoins sa dépendance est moins grande du fait qu'elle est capable de suffire à ses besoins, de gagner sa vie, mais grâce à un métier réputé très féminin : elle fabrique des fleurs artificielles, dans le roman André (1835).

Avec Lélia s'opère, me semble-t-il, un renversement des clichés romanesques dont George Sand avait su se servir jusque là pour décrire la société de son temps et par conséquent la critiquer. Lélia est une héroïne a-typique; ce roman philosophique met en scène une femme forte, et cette force de Lélia s'affirme encore davantage lorsque dans la version de 1839, elle s'attaque à un mythe masculin par excellence : Don Juan. À partir de Lélia, pourrait-on dire en généralisant peut-être un peu trop, mais je ne fais qu'indiquer les grandes lignes, les héroïnes deviennent de fortes femmes qui dirigent l'action des autres personnages, au lieu de la subir. Elles semblent alors - même lorsque la romancière les situe dans un univers très réel - relever de deux figures mythiques de la force et de l'indépendance : Diane et Isis. Pour citer un roman peu connu, mais que la belle édition de Catherine Mariette ${ }^{7}$ nous permet de redécouvrir : dans Simon, Fiamma est une amazone, elle aime la chasse, elle est une excellente cavalière, elle est fort indépendante et pendant longtemps refuse le mariage. Sur un tout autre registre, non plus aristocratique comme l'était Fiamma, mais populaire et même ouvrier, l'héroïne de La Ville Noire est une femme forte qui organise à elle seule la cité coutelière de Thiers; elle aussi retarde beaucoup le moment où elle consent au mariage. Ce thème du mariage différé n'est pas seulement un moyen de suspendre le récit, de tenir en éveil l'attente du lecteur, il est aussi chez les personnages féminins un mode de refus de la servitude.

Tonine ne consent au mariage que lorsqu'elle est devenue, grâce à l'éducation, l'égale de son mari - éducation d'autodidacte, femme et ouvrière, elle ne pouvait en avoir d'autre. «L'horizon de Tonine s'était agrandi. Elle avait voulu entendre de son mieux la science et les arts de l'industrie qu'elle avait à gouverner, et, sans être sortie de son Val-d'Enfer, elle s'était mise au courant du mouvement industriel et commercial de la France ${ }^{8}$. » Tonine sera donc chef d'industrie, pleinement capable. On est loin de l'indolente Indiana.

7. George Sand, Simon, éd. Catherine Mariette, dans Béatrice Didier (dir.), Euvres complètes, Paris, Champion, 2010.

8. George Sand, La Ville Noire, texte établi, préfacé et annoté par Jean Courrier, Montréal, Éditions de l'Aurore, 1989, p. 141. 
Cette transformation progressive du personnage romanesque correspond à une évolution de la romancière. La transformation de son moi n'apparaît nulle part plus nettement que dans la correspondance avec Flaubert, nettement postérieure à Histoire de ma vie, prolongement de l'écriture du moi à deux voix. Dans cet étonnant dialogue du masculin et du féminin, Flaubert l'appelle « cher maître » (bien évidemment, le mot au féminin aurait eu un autre sens); la différence sexuelle ne disparaît certes pas, mais elle est sublimée, dépassée dans la création. Et la création non seulement de deux romanciers, mais de deux épistoliers, et même de deux identités. On relira la lettre du 17 janvier 1869, si importante, me semblet-il. George Sand y formule admirablement le lien de complémentarité qui existe entre Flaubert et elle : « Nous sommes, je crois, les deux travailleurs les plus différents qui existent. Mais puisqu'on s'aime comme ça, tout va bien ${ }^{9}$. L La complémentarité du masculin et du féminin est donc transcendée en quelque sorte dans la création de deux « travailleurs». George Sand a pris par rapport à son moi comme une distance :

L'individu George Sand se porte bien, savoure le merveilleux hiver qui règne en Berry, cueille des fleurs, signale des anomalies botaniques intéressantes, coud des robes et des manteaux pour sa belle-fille, des costumes de marionnettes, découpe des décors, habille des poupées, lit de la musique ${ }^{10}$.

Les activités considérées comme féminines ne nuisent en rien à la création $\mathrm{du}$ « vieux troubadour retiré des affaires qui chante de temps en temps sa petite romance à la lune ${ }^{11} »$. En fait de «petite romance », elle écrit sans cesse pendant cette période, comme tout au long de sa vie. Le «maitre » est un « troubadour », c'est aussi le nom que lui donne Flaubert, et là encore on voit que le masculin est utilisé sans complexe. Sand a une énorme activité non seulement de romancière - « Je fais mon petit roman de tous les ans »-mais aussi de dramaturge, et pas seulement à Nohant : la suite de la lettre annonce que L'Autre va être représentée à l'Odéon.

Toujours dans cette lettre, où elle parle d'elle comme de «l'individu nommé George Sand », elle est amenée curieusement à parler de son passé au masculin : « Il a eu la bêtise d'être jeune, mais comme il n'a point fait de mal, ni connu les mauvaises passions, ni vécu pour la vanité, il a le bonheur d'être paisible et de s'amuser de tout. » Ce masculin découle, certes, du neutre « l'individu », nécessité grammaticale; il n'en est pas moins étonnant quand il s'agit d'évoquer la période de la vie de George Sand où elle est considérée comme le plus femme et même, à en croire la rumeur publique toujours avide d'anecdotes, femme fatale. L'utilisation du masculin devient

9. Gustave Flaubert, George Sand, Correspondance, éd. Alphonse Jacobs, Paris, Flammarion, 1981, p. 213.

10. Ibid., p. 212.

11. Ibid. 
alors un signe de la distanciation à l'égard de soi, à l'égard aussi de l'image d'elle-même qu'on a voulu donner.

Le féminin réapparaît pour condamner l'horreur de la guerre, le 15 août 1870 : « Il y a toujours une femme dans la peau du vieux troubadour. Cette boucherie humaine met mon cœur en loques. Je tremble aussi pour tous mes enfants et amis qui vont se faire hacher. Et, au milieu de tout cela, pourtant mon âme se relève et a des élans de foi ${ }^{12}$. » Et l'écriture ne s'interrompt pas. Césarine Dietrich vient d'être achevé : « J'ai fini un roman au milieu de cette tourmente, me hâtant pour n'être pas brisée avant la fin. Je suis lasse comme si je m'étais battue avec nos pauvres soldats ${ }^{13}$. » Les opinions politiques de Flaubert et de Sand diffèrent encore plus que leurs méthodes littéraires, mais cela ne les empêche pas de s'aimer. Flaubert est pessimiste. Sand ne peut s'arrêter d'espérer en l'avenir :

[J]e ne sais pas si tu es de mon avis, que la liberté pleine et entière, nous sauverait de ces désastres et nous remettrait dans la voie du progrès possible. Les abus de liberté ne me font pas peur par eux-mêmes mais ceux qu'ils effraient penchent toujours vers les abus de pouvoir ${ }^{14}$.

Malgré des moments de dépression, d'incompréhension devant les événements et la Commune, malgré des lettres qui trahissent l'angoisse dans cette période si troublée de 1870-1871, l'impression dominante est bien, du côté de G. Sand, un étonnant équilibre qui provient en grande partie de ce que les clichés du masculin et du féminin de son époque faiblesse et passivité féminine, force créatrice de l'homme - clichés qui ont la vie dure et se poursuivront au $\mathrm{Xx}^{\mathrm{e}}$ siècle, sont totalement dépassés par elle. Cette correspondance avec Flaubert exprime ce dépassement et peut-être même a contribué à l'affirmer précisément parce qu'il s'agissait d'une correspondance entre un homme et une femme, un dialogue dans lequel les rôles peuvent être inversés : George Sand représentant souvent la force du «maître », de la conseillère maternelle, et Flaubert se sentant faible et malade, souvent démuni et dans une relation filiale. Ce rapport a priori curieux correspond probablement à une réalité, mais il est fortement orchestré par ce que l'on pourrait appeler l'effet correspondance : à s'exprimer dans la lettre la force ou la faiblesse se confirment. Et Sand de recourir à une image de fécondation qui, en étant celle de la pluie, évite d'être exclusivement masculine, grâce au genre du mot «pluie »: " Tes lettres tombent sur moi comme une pluie qui mouille, et fait pousser tout de suite ce qui est en germe dans le terrain ${ }^{15}$. »

12. Gustave Flaubert, George Sand, op. cit., p. 308.

13. Ibid., p. 308-309.

14. Ibid., p. 325.

15. Ibid., p. 356. 
Le dépassement des clichés du masculin et du féminin est possible chez Sand grâce à une acceptation du temps et de son corps, grâce à une intense communion avec les forces de la nature, et surtout grâce à la création littéraire dont elle est persuadée qu'elle est « androgyne». Elle a ce propos étonnant dans une lettre du 23 juillet 1871 - donc à soixante-sept ans :

Le jour où j'ai résolument enterré ma jeunesse, j'ai rajeuni de vingt ans. Tu me diras que l'écorce n'en subit pas moins l'outrage du temps. Ça ne me fait rien, le cœur de l'arbre est fort bon et la sève fonctionne comme dans les vieux pommiers de mon jardin qui fructifient d'autant mieux qu'ils sont plus racornis ${ }^{16}$.

Dans cette métaphore des pommiers s'expriment à la fois l'acceptation du temps, l'identification à la nature, et la créativité : la romancière donne des romans, comme les vieux pommiers des pommes, avec la régularité des saisons - au moins un roman chaque année.

Masculin/féminin? il ne s'agit donc plus exactement d'abolir cette différence, mais d'être tout à la fois homme et femme; cela ne supprime pas la différence, cela permet, grâce à l'équilibre du «moi », grâce à la création androgyne, d'acquérir des propriétés que la société a tort de vouloir dissocier; non pas être l'autre, mais être une totalité, et aider l'autre, qu'il soit le correspondant ou le lecteur, à devenir lui aussi cette totalité.

\author{
Béatrice Didier \\ École normale supérieure
}

16. Ibid., p. 339. 